\title{
ADVANCES IN REDUCING MICROLEAKAGE IN DENTAL COMPOSITES
}

\author{
Hai-Huan GONG, ${ }^{\text {a }}$ Xiao-Meng ZHANG, ${ }^{\text {a }}$ Xiao-Wei GUO,${ }^{\text {a }}$ Ping GAO, ${ }^{\text {a }}$ Qi ZHAO, ${ }^{\text {a }}$ Tong XU, ${ }^{\text {a }}$ \\ Xiao-Yu SHI, ${ }^{b}$ Song ZHU ${ }^{a^{*}}$ and Zhan-Chen CU ${ }^{b^{*}}$ \\ ${ }^{a}$ Department of Prosthetic Dentistry, School and Hospital of Stomatology, Jilin University, Changchun 130021, P.R. China \\ ${ }^{\mathrm{b}}$ State Key Lab of Supramolecular Structure and Materials, College of Chemistry, Jilin University, Changchun 130021, P.R. China
}

The clinical success of esthetic restorations, including composite resins, depends on the quality and durability of the interface between the tooth and restoration. Current commercially available materials do not possess ideal sealing at the marginal interfaces, and marginal microleakage leads to clinically significant issues for patients and clinicians. Many factors may affect microleakage formation, including the adaptation of composite resins to the tooth surface, the adhesive system used, and the polymerization shrinkage of the materials. The evolution of restorative materials, designed to achieve low shrinkage and improve mechanical properties, could lead to restorations with improved clinical

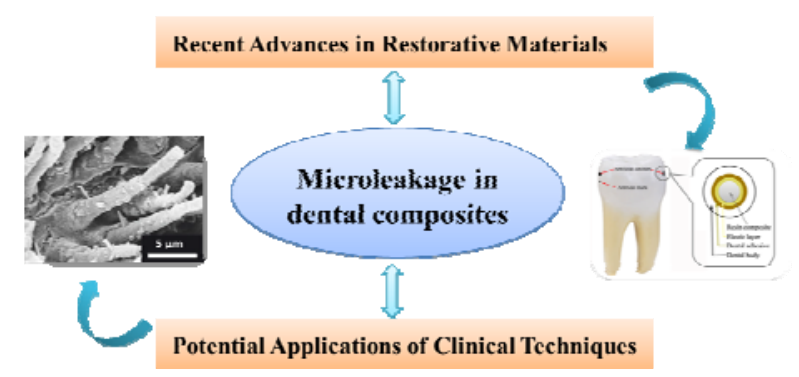
performance. Furthermore, searching for clinical techniques to optimize the integrity of resin/dentin bonds is significant, and the evaluation of microleakage is also critical to the assessment of restorative materials. The objective of this paper was to review the literature on the recent advances in restorative materials, the potential applications of clinical techniques, and the methods of measuring microleakage.

\section{INTRODUCTION}

Since the 1960s, composite resins have been extensively used to restore carious lesions, tooth wear, coronal fractures, and congenital tooth defects because of their desirable esthetics, minimally invasive preparation technique, excellent physical and chemical properties, and ease of handling. ${ }^{1,2}$ They have almost completely replaced dental amalgam, and have become the first choice for a direct restorative material. ${ }^{3}$ However, the optimal performance of composite resin restorations depends on the proper polymerization of the resin component, which is accompanied by a reduction in the material's volume, ${ }^{4}$ or polymerization shrinkage. Despite various developments in composite resin materials over recent years, polymerization shrinkage and its related stress remain their greatest drawback and lead to clinical problems. ${ }^{5,6}$ The marginal adaptation of the restoration to the tooth preparation might be influenced by the volume change of the composite resin. ${ }^{3}$ A consequence of stress due to polymerization shrinkage causes the formation of internal voids and marginal gaps between the restorative material and tooth structure. ${ }^{4-6}$ Tooth demineralization can occur at these gaps because of the leakage of either bacteria or their acid by-products, termed microleakage. ${ }^{7}$

The term "microleakage," still used today, appeared in IADR meeting abstracts from $1959^{8}$ and is defined as a channel between the cavity wall and restorative material that a series of substances, including bacteria, liquids, molecules, and ions, can penetrate but that cannot be directly detected clinically. ${ }^{9}$ Microleakage occurs more readily on

\footnotetext{
*Corresponding author: zhusong1965@163.com
} 
the proximal gingival margins of class II restorations, in which little or no enamel remains, especially those undergoing mechanical loading. ${ }^{7}$

Microleakage is considered the main factor affecting the longevity and durability of restorations because of marginal staining and the acceleration of restoration breakdown, tooth hypersensitivity, secondary caries, and pulp injury. ${ }^{9}$ Furthermore, clinical studies have shown lower failure rates and better longevity for amalgams compared with those of composite restorations. ${ }^{10-13}$ If the indirect restoration technique for composite resins is applied to posterior teeth, the shrinkage caused by direct filling during the curing of the resins is effectively reduced, and the degree of polymerization and marginal adaptation of the resins are significantly increased; however, secondary caries and the fracture of indirect composite resin restorations may still occur. ${ }^{14}$ One of the main reasons is the different coefficient of thermal expansion between the composite resins (about 18 to $24 \times 10^{-6} /{ }^{\circ} \mathrm{C}$ and dentin and enamel about 11 to $\left.17 \times 10^{-6} /{ }^{\circ} \mathrm{C}\right) .{ }^{15}$ Earlier studies have demonstrated that the greater the difference in the coefficient of thermal expansion between the composite resins and dental hard tissue, the more microleakage is likely to occur. ${ }^{16}$

Adequate marginal integrity between the tooth and composite resin restoration is needed to increase the durability of the restoration. Consequently, the developments of new materials and different clinical techniques have been aimed at decreasing microleakage, making this an important topic for researchers and clinicians. Therefore, this review article will focus on recent advances in restorative materials, potential applications of clinical techniques, and methods for measuring microleakage, starting with a discussion of selected composite restorative materials and techniques that are used clinically to reduce microleakage in composite resin restorations.

\section{Recent Advances in Restorative Materials Polymerization shrinkage and microleakage}

Microleakage may be the result of the adaptation of composite resins to the tooth surface, the adhesive system selected, and the polymerization shrinkage of the materials used. The polymerization of a resin component is characterized by transforming monomer molecules into a polymer network, which occupies less volume than the monomers. ${ }^{4}$ Polymerization shrinkage stress increases rapidly for all composite resins during two periods of time, the first during the initial $10 \mathrm{~s}$ of light curing and the second by a quite slow increase until the end of light curing. ${ }^{17}$ During polymerization, the van der Waals forces between the monomers are changed into covalent bonds, reducing the distance between the monomers and leading to a reported volume contraction in the range of $2 \%$ to $5 \% .^{17,18}$ This polymerization shrinkage creates contraction stress at the interface between the teeth and the restoration, resulting in marginal gap formation and debonding when the bond strength is less than the shrinkage stress. The gaps formed due to polymerization shrinkage are only a few micrometers in width but may extend throughout the wall interface and eventually cause microleakage. $^{19}$

Different composite resins, adhesive systems, and clinical techniques have been developed to minimize microleakage in adhesive-restorative procedures and improve their clinical effects. Based on the deficiencies in the current materials and the relationship between polymerization shrinkage and microleakage mentioned above, new composite resins with lower polymerization shrinkage, contraction stress, water sorption, and better mechanical properties have been developed. Meanwhile, new types of polymer matrix materials different from conventional acrylic monomers have been reported.

\section{New low shrinkage matrix}

In recent years, oligomers which form classic thiol-ene reactions have been shown to promote homogeneous methacrylate network formation, increase the degree of conversion and improve mechanical properties. ${ }^{20}$ Bacchi et al. have found that incorporating thio-urethane oligomers into composite cements can effectively improve properties. $^{21,22}$ Incorporating thio-urethane oligomers into methacrylate networks has also been shown to effectively improve the properties of methacrylatebased composite resins because of the chaintransfer reactions that delay polymer vitrification, while at the same time resulting in increasing the degree of conversion ${ }^{23}$ and mechanical properties, particularly toughness and fracture toughness. ${ }^{21}$ In the specific case of oligomer additives, the lower volumetric shrinkage could be obtained because of the high molecular weight of additives. ${ }^{22,24}$

The allyl sulfide functional group has been incorporated into the norbornene-methacrylate resin system by Park et al., who used addition- 
fragmentation chain transfer (AFCT) to relax shrinkage stress and create adaptable polymer networks. ${ }^{25} \mathrm{AFCT}$ functional groups were shown to undergo bond breaking and reforming as the same structure throughout the resin polymerization, resulting in the rearrangement of network strands. Another, ring-opening (allyl sulfide-containing ring) reaction also plays an important role in reducing volume shrinkage. ${ }^{25}$ Additionally, the AFCT of allyl sulfide does not essentially reduce the net crosslinking density. As a result, the use of monomers with AFCT can facilitate stress relaxation and dissipation and, ${ }^{25,26}$ accommodate and reduce polymerization stress, while maintaining mechanical properties comparable with those of traditional glassy networks.

\section{Bulk-fill composite resins}

Recently, bulk-fill composite resins have been introduced with less filler content, larger filler size, and higher translucency than conventional composite resins. ${ }^{27}$ The principle is based on the incorporation of a new type of high molecular weight matrix monomer into a conventional resin matrix and the use of modified nanofiller. ${ }^{28}$ The advantage of bulk-fill composite resins is the claim that it can be placed in 4-mm increments, allowing for adequate cure in one step in contrast with the current maximum 2-mm incremental placement technique, without any adverse effects on polymerization shrinkage or shrinkage stress. ${ }^{28,29}$ Also, they are suitable for use in uncooperative patients because they are easy to handle.

Scotti et al. used low-viscosity bulk-fill composite resin and nanohybrid composite resin to fill the pulp chamber after root canal treatment. They reported that the bulk-fill composite resin had better marginal adaptation and could reduce the occurrence of pulp floor fissures. ${ }^{30}$ Poggio et $a l$. and Mosharrafian et al. evaluated microleakage in class II conventional and bulk-fill composite resin restorations and reported that all composite resin restorations showed some degree of microleakage, but that SonicFill bulk-fill composite resin showed the lowest microleakage.

${ }^{31}$ Alkhudhairy et al. found that bulk-fill composite resins had better marginal sealing than fiberreinforced composite resins for restoring class II cavities of premolar teeth. ${ }^{32}$ However, Rengo et al. found no significant difference in microleakage when three conventional composite resins were used to restore class II cavities with the incremental filling technique and when class II cavities were restored with bulk-fill composite resins in permanent teeth. ${ }^{33}$

None of these bulk-fill composite resins have been subjected to long-term clinical trials since the materials are too new; therefore, long-term studies are needed to evaluate the marginal adaptation of bulk-fill composite resins. Future research on the application of new techniques is recommended for a more accurate assessment of microleakage.

\section{Flowable composite resins}

Flowable composite resins are conventional composite resins that have been formulated to have greater fluidity before light curing and have been available since the mid-1990s. ${ }^{34}$ Flowable composite resins are reported to provide better marginal adaptation than traditional composite resins, ${ }^{35}$ and their lower modulus of elasticity enables them to absorb polymerization shrinkage stress ${ }^{36}$ and decrease microleakage at the interface. Malmstrom et al. suggested that a 2.0-mm layer of flowable composite resin improves marginal adaptation more than a $0.5-\mathrm{mm}$ thickness in class II restorations, ${ }^{37}$ Kwon et al. found that flowable composite resins exhibited a resin tag formation performance similar to that of traditional pit and fissure sealants. $^{38}$

However, flowable composite resins have a higher resin matrix proportion than traditional composite resins. As the degree of polymerization shrinkage is influenced by the quantity of resin matrix, flowable composites shrink more while polymerizing and contract more at the interface compared with conventional composite resins, suggesting a higher possibility of microleakage. Some have reported that flowable composite resins have shown a trend towards marginal discrepancy of the restorations after thermocycling due to their high coefficient of thermal expansion. ${ }^{39}$

Flowable composite resins are novel conventional materials compared with conventional composite resins and could be developed into an inhomogeneous group of materials with various applications. As a consequence, clinicians should be aware of the variety in their mechanical and physical properties ${ }^{40}$ when selecting the most appropriate material for a particular clinical situation. Further studies are needed to investigate microleakage interactions and evaluate flowable composite resins in vivo. 


\section{Polyurethane elastomer as an elastic layer}

Even if the polymerization shrinkage of the composite resins approaches $0 \%$, which eliminates the presence of a marginal gap between dental hard tissue and composite resins after light curing, a gap will eventually appear and will be aggravated by temperature changes in the oral environment. This is because of the different thermal expansion coefficients of the composite resin and dental hard tissues. ${ }^{41}$

To overcome the thermal expansion mismatch, a new strategy by using a polyurethane (PU) elastic layer to absorb interface stress has been put forward by Zhang et al. for overcoming microleakage. ${ }^{41} \mathrm{PU}$ is a type of multifunctional material with various applications. The main substrates of polyurethane are the so-called flexible segments and rigid segments, which consist of polyol and isocyanate, respectively. By modulating the type and molar ratio of components, materials can be obtained with optimal properties including flexibility or rigidity and whether thermoplastic or waterborne. ${ }^{42}$ The specific adjustable mechanical, physical, biological, and chemical properties of PU are attracting the attention of researchers who wish to adapt them for different applications.

Zhang et al. introduced a photo-cured PU elastic layer in a dental composite resin restoration.
The structure of this new strategy is illustrated in Figure 1. Originally, the connection of traditional acrylic resin materials and tooth tissues is a rigid bonding (i.e., composite resin-adhesive-tooth). The adhesive layer is under the strongest stresses during the acrylate material's curing process and during transient thermal and mechanical loading, the new concept based on the photo-cured PU elastic layer may change the interface into a flexible bond (i.e., composite resin-elastomeradhesive-tooth), which may effectively buffer the stresses and make the bonding interface more stable and durable. As a result, the application of PU elastomer might decrease the occurrence of microleakage (Fig. 2) and improve the durability and service life of composite resins. The mechanical properties, the restorative effect, and the cytotoxicity of this polyurethane elastic layer material have been tested, with favorable preliminary results. This study indicates a new direction for researchers in that an intermediate elastic layer between the restorative materials and the cavity walls could absorb a large part of shrinkage stress and stress caused by thermal expansion. This may be a better method of reducing microleakage than focusing on improving the mechanical properties of materials.

(A)

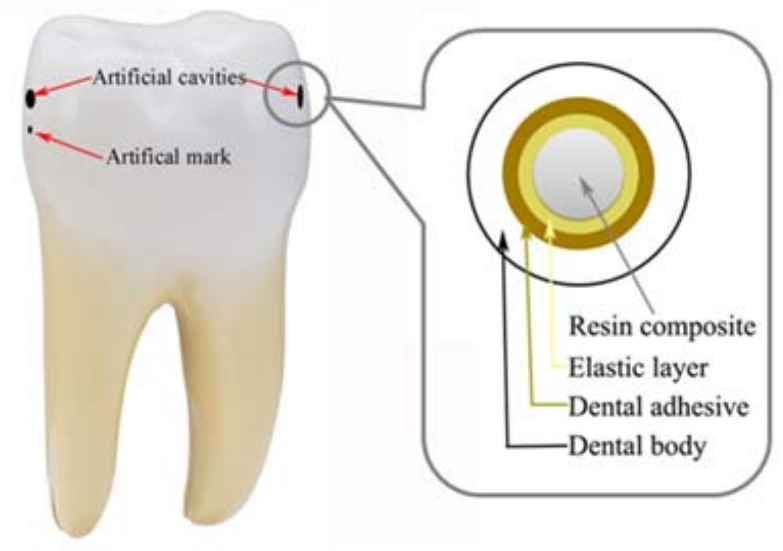

(B)

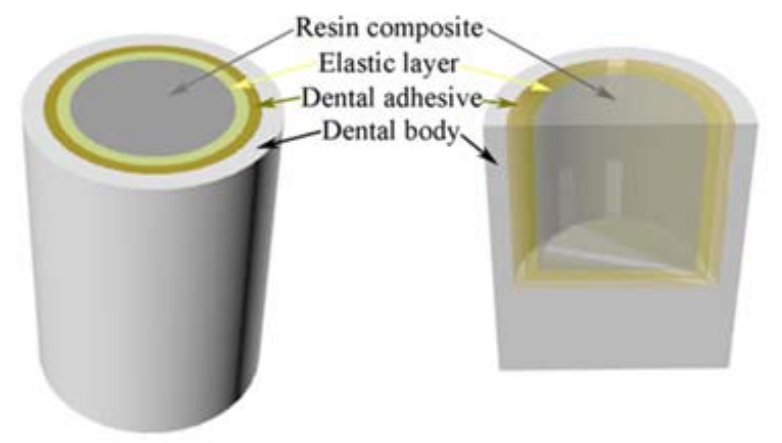

Fig. 1 - schematic model for the elastic layer property test specimen (scale is not important and is not given here); B the structure of the artificial cavities restored with the elastic layer (Adapted from Zhang et al. 2015, with permission). 


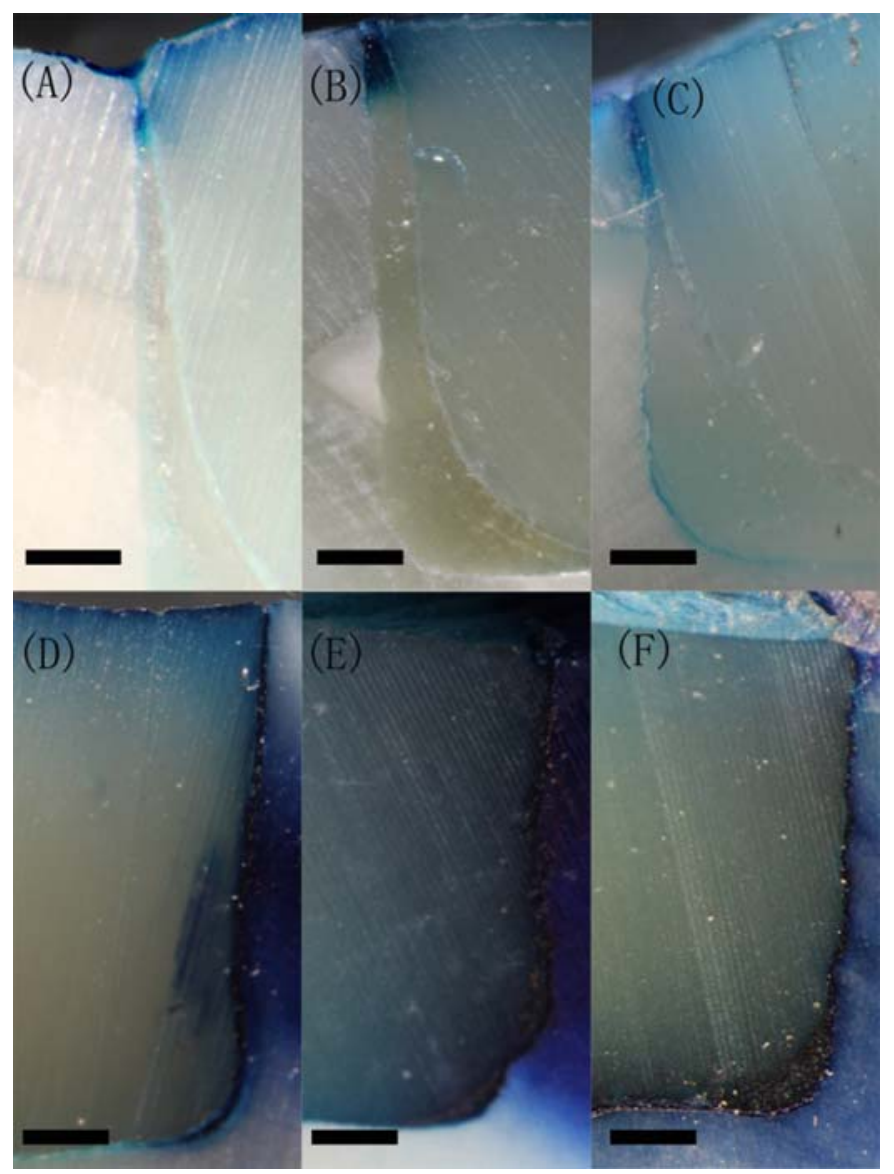

Fig. 2 - The tooth sections that had been soaked for 24 hours, (A) and (D); 3 days, (B) and (E); and 7 days, (C) and (F). Scale bar $=300 \mathrm{~mm}$ (Adapted from Yingchao et al. 2015, with permission).
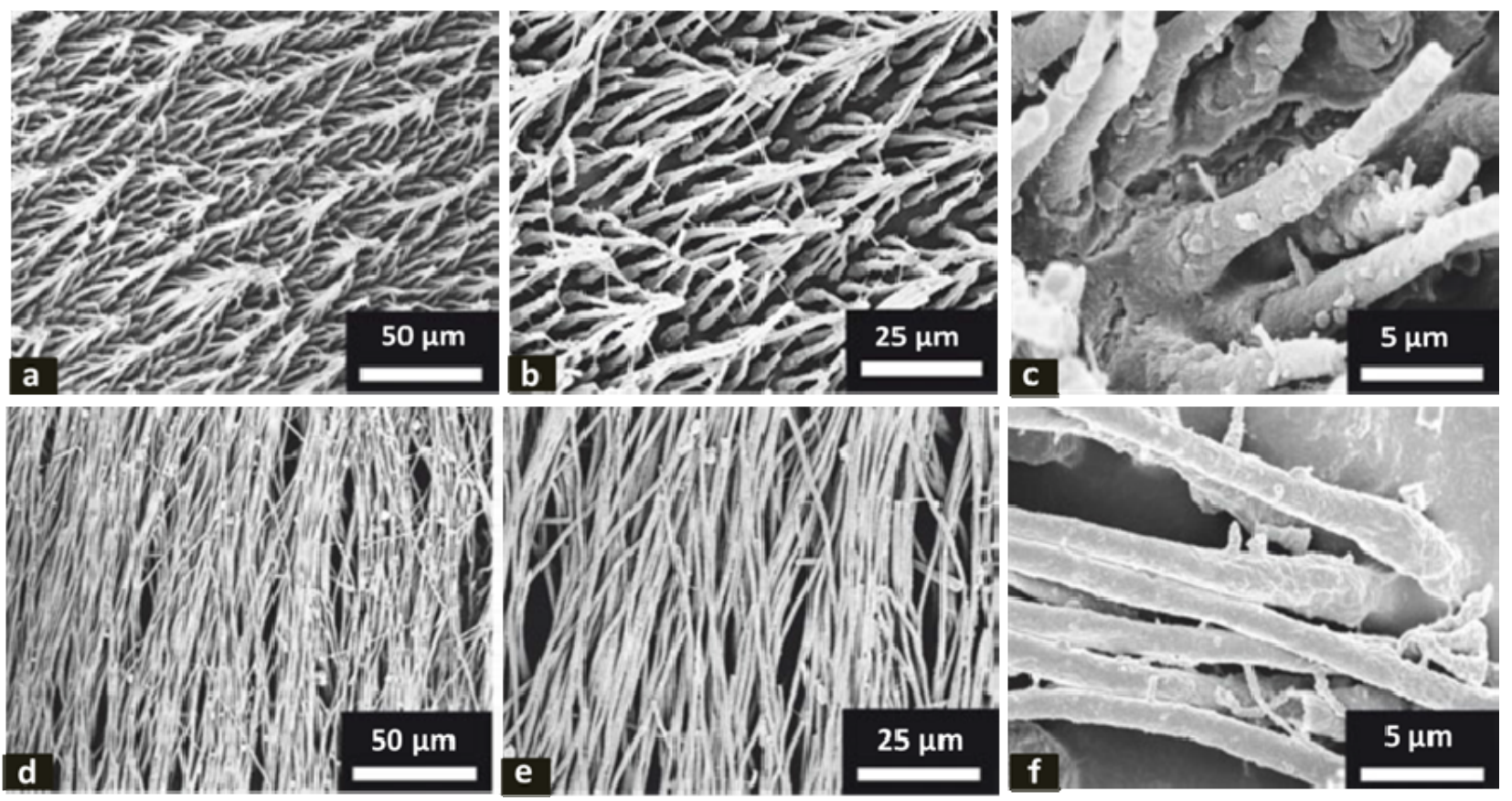

Fig. 3 - Representative SEM images of resin tags formed in the control and high-pressure air-blowing groups. a-c:SEM micrographs indicate typical resin tag formed at the Single Bond/dentin interface. The resin tags are shorter and become thinner as they traverse from the occlusal to the pulpal end of the resin tag. d-f: SEM micrographs of resin tags formed at the bonding interface when Single Bond adhesive was applied with high-pressure air blowing. Dense, long, homogenous resin tags covered the basal part of the bonding interface (d). Under higher magnification, lateral branches of resin tags were observed (arrow) (f) (Adapted from Chen et al. 2014, with permission). 


\section{Potential Applications of Clinical Techniques Cavity preparation with an erbium laser}

Recently, scientists have evaluated the impact of an erbium laser on microleakage. The erbium yttriumaluminum garnet (Er:YAG) laser has been approved as a dental hard tissue laser for clinical application by the United States Food and Drug Administration (FDA). ${ }^{43}$ The $2940-\mathrm{nm}$ wavelength radiation of the Er:YAG laser conforms with the primary absorption peaks of water and hydroxyapatite and cuts by means of a thermochemical reaction with the tooth tissue and the principle of microexplosion. At the microscopic level, because it produces an irregular surface with open dentinal tubules and without a smear layer, the Er: YAG laser is a suitable method for dentin treatment.

Phanombualert et al., researched the microleakage of self-etch adhesive systems in class $\mathrm{V}$ cavities prepared with an Er:YAG laser with different impulse patterns. Compared with diamond rotary instrument groups, the microleakage in the Er:YAG laser emission groups was markedly reduced. Minimum microleakage was associated with an energy density of $3.77 \mathrm{~J} / \mathrm{cm}^{2}$ on both the occlusal and gingival cementum margin. ${ }^{44}$ A similar conclusion was reported for erbium, chromium, yttrium, scandium, gallium, and garnet (Er,Cr:YSGG) used in Class V cavities of primary teeth with nanofilled resinmodified glass ionomer restorations. ${ }^{45}$ However, some studies showed no significant difference in microleakage between cavities prepared by laser (Er:YAG and Er,Cr:YSGG) and diamond rotary instruments . ${ }^{46-48}$

Nevertheless, erbium laser technology showed desirable results for pain-free cavity preparations, and no significant difference was found with respect to restoration loss, pulp vitality, and postoperative sensitivity. ${ }^{49}$ Investigations of laser tooth preparation on microleakage of composite resins have not reported consistent results. More clinical studies should be conducted to observe the clinical behavior of teeth prepared with erbium lasers.

\section{Adhesive displacement with pressure air blowing}

Even though marginal gaps were not visible, Sano et al. observed the penetration of silver nitrate tracer into the hybrid layer and of relatively smaller tracer ions (silver) into the full thickness in some areas, and also into the adhesive resins. If the adhesive does not penetrate, the decalcified area by the acids forms a weak zone that can be easily hydrolyzed, followed by microleakage. ${ }^{50}$ Under such circumstances, nanoleakage must be elimi- nated to decrease microleakage. Chen et al. conducted a series of feasibility studies on a method where high-pressure air is directed during adhesive application. ${ }^{51}$ This new procedure may provide an additional driving force for resin infiltration against pulpal pressure; increasing the amount of resin impregnation within the hybrid layer, which results in longer resin tags (Fig. 3), reduced nanoleakage and provided a more integrated bonding interface. Also, Zhou et al. found that augmented-pressure adhesive displacement created a more complete structure of the adhesive-dentin mixed layer and improved caries resistance. Additionally, when the air pressure applied is equal or lower than $0.3 \mathrm{MPa}$, vital teeth will not suffer irreversible pulpal damage. ${ }^{52}$ These results suggest the clinical application and significance of the pressure air blowing technique in the application of dentin adhesives.

\section{METHODS \\ FOR MEASURING MICROLEAKAGE}

The evaluation of microleakage is a significant index in assessing restorative materials and has been performed in vitro, as an in vivo model is difficult to apply. Techniques used to evaluate the level of interfacial microleakage in vitro include compressed air, chemical, bacteria, radioactive markers, dye penetration, electrochemical investigations, scanning electron microscopy (SEM), and recently X-ray microcomputed tomography (micro-CT) and optical coherence tomography (OCT); the results, however, vary considerably.

\section{Dye-penetration method}

The conventional dye-penetration method has been the one most commonly used in vitro, because microleakage itself is always related to liquidpenetration. Various dye solutions have been used, typically involving fluorescein, methylene blue, silver nitrate solution, fuchsin solution, or anilineblue. After different dwelling times, the sample is sectioned to visually examine dye penetration around the restoration under a microscope. One of the major drawbacks of this method is qualitative assessment by observing the presence or absence of the dye in a particular section. A semi-quantitative result may be achieved by using a length scale, but there are subjective factors which can lead to results that are not comparable. Another drawback of this method is that the results obtained are two-dimensional (2D) in nature. In addition, it is necessary to distinguish between a certain degree of dentin staining and actual microleakage. 


\section{X-ray microcomputed tomography (Micro-CT)}

Without destroying the original specimen, microCT recreates a virtual model with a spatial resolution at a micrometer level by using X-rays to scan cross-sections of a 3D object. Recently, Carrera et al. have developed an approach which quantifies the volume of leakage using micro-CT, silver nitrate permeation, and image segmentation. ${ }^{53}$ In another study, nondestructive micro-CT has been used to analyze the internal adaptation of composite resin to dentin; micro-CT has also been used to evaluate the relationship between internal adaptation and polymerization shrinkage. ${ }^{54}$

However, Zhao et al. ${ }^{55}$ used the micro-CT and dye-penetration method to examine leakage around the same Class $\mathrm{V}$ resin composite restorations. They concluded that micro-CT has accuracy comparable with that of the conventional dye-penetration method in terms of measuring leakage in the dentin region but offers inferior accuracy in the enamel region as a result of the lower radiopacity contrast of silver tracer to enamel (Fig. 4). The same conclusion was reached by Jacker-Guhr et al., ${ }^{56}$ who suggested that to better utilize micro-CT, a new microleakage tracer which would distinguish well between dental tissue and materials would be necessary. Micro-CT is not yet an absolutely reliable method of assessing the marginal integrity of dental restorations.
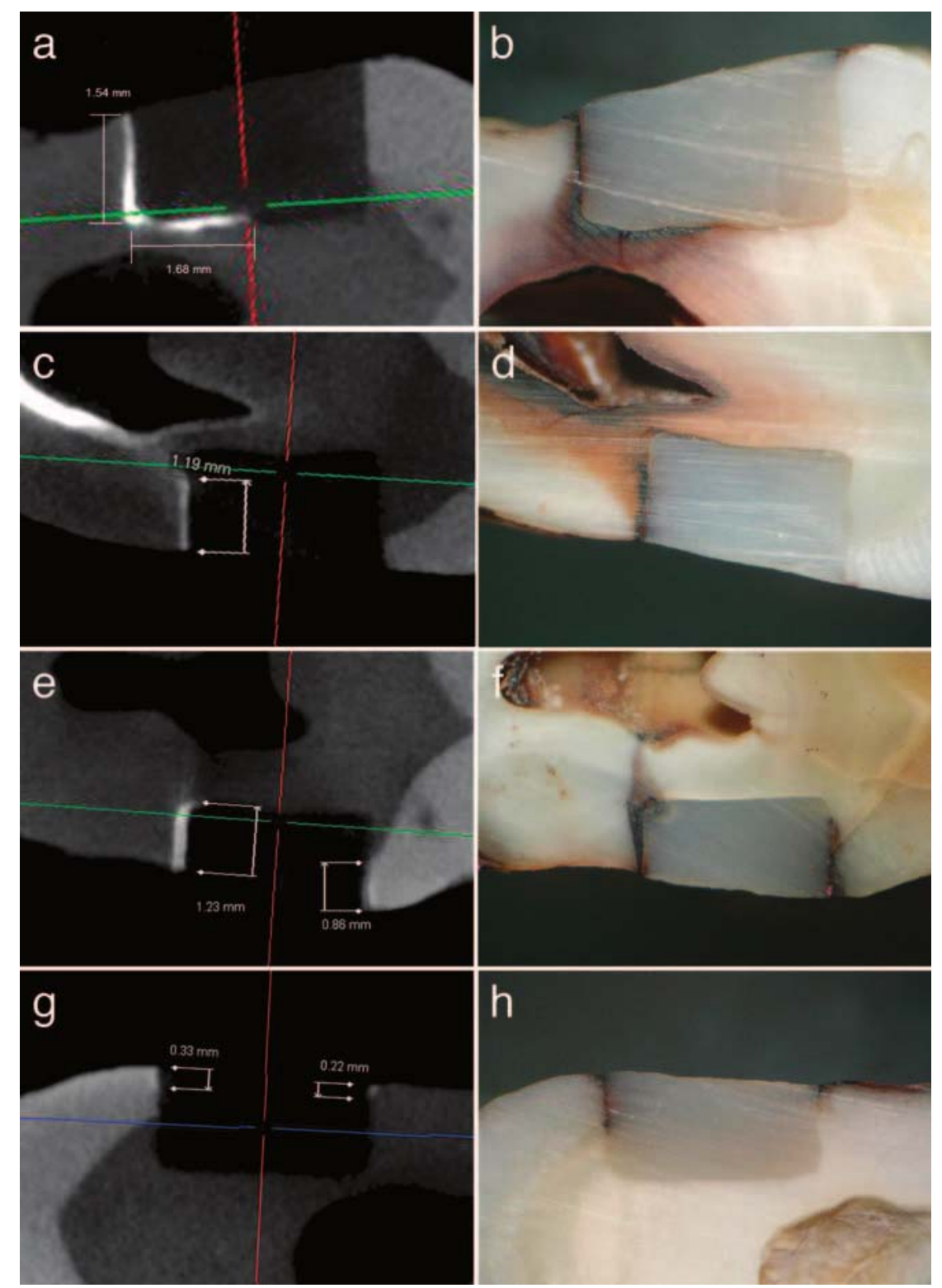

Fig. 4 - Marginal leakage on the middle longitudinal sections of four restorations. (a, c, e, and g) Images of the micro-CT; (b, d, f, and h) corresponding images from the microscope after cutting (Adapted from Zhao et al. 2014, with permission). 


\section{Optical coherence tomography (OCT)}

Optical coherence tomography (OCT) is a relatively new, completely nondestructive, and powerful tool. It has great prospects in dentistry and may provide a good method for the diagnosis of microleakage. OCT offers high resolution and a penetration depth of up to a few millimeters and does not need specimen preparation or vacuum conditions. Additionally, it provides real-time restoration observation without X-ray radiation exposure. In 1991, Huang et al. first reported on OCT, which has since been widely used in clinical applications. ${ }^{57}$ OCT is an advanced tool that uses a microscale cross-section imaging technique to adopt near low-coherence interferometry; the principle is to convert the backscattered light into a signal intensity that is displayed as an image after a laser beam is projected across a restoration. ${ }^{58}$

Swept-source optical coherence tomography (SS-OCT) is a type of OCT. It has a high axial resolution of about $10 \mu \mathrm{m}$, which provides sufficient information for dental clinical applications such as the detection of cracks and microleakage. One study ${ }^{59}$ described microleakage sensing based on (SS-OCT), where the calculated length was $401 \mu \mathrm{m}$ and the width was $148 \mu \mathrm{m}$, which is not significantly different from histological biopsy measurements. In addition, the authors compared various methods currently used in general dentistry for diagnosis.

Reproducibility is essential when evaluating microleakage so that the research can be considered reliable. The diagnosis of microleakage can help prevent secondary caries in clinical treatment plans. Further research on internal adaptation and microleakage will therefore be especially important.

\section{CONCLUSIONS}

Research in the last few years has advanced the assessment of microleakage, improved restorative materials, and developed effective techniques for decreasing microleakage. However, the reduction of microleakage has not been accomplished: 1) effective, widely accepted approaches are still lacking, whereas controversial methods abound; 2) holistic, multitarget approaches are few, whereas the majority of methods focus on single factor; 3) most of the technologies are reported in the basic research stage, and few have advanced to clinical applications; 4) most studies are focused on the short term, and studies with long follow-up periods are rare.

Acknowledgements: This work was supported by the National Natural Science Foundation of China (NSFC, Grant No. 81671033). We are grateful to editor Enid Rosenstiel for revising the manuscript critically. The authors declare no potential conflicts of interest with respect to the authorship and/or publication of this article.

\section{REFERENCES}

1. S. Mosharrafian, A. Heidari and P. Rahbar, J. Dent. (Tehran, Iran), 2017, 14, 123-130.

2. Y. Kwon, J. Ferracane and I.-B. Lee, Dent. Mater, 2012, 28, 801-809.

3. I. Nedeljkovic, W. Teughels, J. De Munck, B. Van Meerbeek and K. L. Van Landuyt, Dent. Mater, 2015, 31, e247-e277.

4. C. J. Soares, M. P. Rodrigues, A. B. F. Vilela, A. L. FariaE-Silva ,C. S. Pfeifer, D.Tantbirojn and A. Versluis, Braz. Oral Res., 2017, 31(suppl 1), e62.

5. C. J. Soares , A. A. Bicalho, C. Verissimo , P. Soares, D. Tantbirojn and A.Versluis, Oper. Dent., 2016, 41, 491500.

6. C. M. Rosatto, A. A. Bicalho, C. Veríssimo, G. F. Bragança, M. P. Rodrigues, D. Tantbirojn, A. Versluis, C. J. Soares, J. Dent., 2015, 43, 1519-1528.

7. J. L. Ferracane, J. Dent. Res., 2017, 96, 364-371.

8. A. Jokstad, Dent.Mater, 2016, 32, 11-25.

9. M. Radhika, G. S. Sajjan, B. Kumaraswamy and N. Mittal, J. Conserv. Dent., 2010, 13, 9-15.

10. K. Sunnegårdh-Grönberg, J. W. van Dijken, U. Funegård, A. Lindberg and M. Nilsson, J. Dent., 2009, 37, 673-678.

11. J. A. Soncini, N. N. Maserejian, F. Trachtenberg, M. Tavares and C. Hayes, J. Am. Dent. Assoc., 2007, 138, 763-772.

12. S. E. Kopperud, A. B. Tveit, T. Gaarden, L. Sandvik and I. Espelid, Eur. J. Oral. Sci. ,2012, 120, 539-548.

13. Y. J. Rho, C. Namgung, B. H. Jin, B. S. Lim and B. H. Cho, Oper. Dent., 2013, 38, 572-582.

14. C. D'Arcangelo, M. Zarow, F. De Angelis, M. Vadini, M. Paolantonio, M. Giannoni and M. D'Amario, Clin. Oral Investig., 2014, 18, 615-624.

15. A. Alnazzawi and D. C. Watts, Dent. Mater, 2012, 28, 1240-1249.

16. R. H. Bullard, K. F. Leinfelder and C. M. Russell, J. Am. Dent. Assoc., 1988, 116, 871-874.

17. R. J. Kim, Y. J. Kim, N. S. Choi and I. B. Lee, J. Dent., 2015, 43, 430-439.

18. C. J. Kleverlaan and A. J. Feilzer, Dent. Mater., 2005, 21, 1150-1157.

19. E. Karaman and G. Ozgunaltay, Oper Dent., 2014, 39, 325-331.

20. C. E. Hoyle and C. N. Bowman, Angew. Chem. Int. Ed. Engl., 2010, 49, 1540-1573.

21. A. Bacchi, A. Dobson, J. L. Ferracane, R. Consani and C. S. Pfeifer, J. Dent. Res., 2014, 93, 1320-1325.

22. A. Bacchi, R. L. Consani, G. C. Martim and C. S. Pfeifer, Dent. Mater, 2015, 31, 565-574.

23. K. A. Berchtold, T. M. Lovestead and C. N. Bowman, Macromol., 2002, 11, 729-738.

24. A. Bacchi, M. Nelson and C. S. Pfeifer, Dent. Mater, 2016, 32, 233-239. 
25. H. Y. Park, C. J. Kloxin, A. S. Abuelyaman, J. D. Oxman and C. N. Bowman, Dent.Mater., 2012, 28, 1113-1119.

26. H. Y. Park, C. J. Kloxin, M. F. Fordney and C. N. Bowman, Dent. Mater., 2012, 28, 888-893.

27. N. Ilie, S. Bucuta and M. Draenert, Oper. Dent., 2013, 38, 618-625.

28. A. F. Reis, M. Vestphal, R. C. D. Amaral, J. A. Rodrigues, J. F. Roulet and M. G. Roscoe, Braz. Oral Res., 2017, 31, $37-48$.

29. H. El-Damanhoury and J. Platt, Oper. Dent., 2014, 39, 374-382.

30. N. Scotti, C. Alovisi, A. Comba, G. Ventura, D. Pasqualini, F. Grignolo and E. Berutti, J. Endod., 2016, 42, 160-163.

31. C. Poggio, M. Chiesa, A. Scribante, J. Mekler and M. Colombo, Med. Oral. Patol. Oral Cir. Bucal., 2013, 18, e793-e798.

32. F. I. Alkhudhairy and Z. H. Ahmad, J. Contemp. Dent. Pract.,2016, 17, 997-1002.

33. C. Rengo, G. Spagnuolo, G. Ametrano, C. Goracci, A. Nappo, S. Rengo and M. Ferrari, Int. J. Adhes. Adhes., 2015, 60, 123-129.

34. K. Baroudi and J. C. Rodrigues, J. Clin. Diagn. Res., 2015, 9, ZE18-24.

35. J. Sabbagh, J. Vreven and G. Leloup, Dent. Mater, 2002, $18,64-71$

36. A. Szesz, S. Parreiras, E. Martini, A. Reis and A. Loguercio, J. Dent., 2017, 65, 11-21.

37. H. S. Malmstrom, M. Schlueter, T. Roach and M. E. Moss, Oper. Dent., 2002, 27, 373-380.

38. H. B. Kwon and K. T. Park, Pediatr. Dent., 2006, 28, 48-53.

39. N. M. Hernandes, A. Catelan, G. P. Soares, G. M. Ambrosano, D. A. Lima, G. M. Marchi, L. R. Martins and F. H. Aguiar, J. Investig. Clin. Dent., 2014, 5, 283-288.

40. S. Rahimian-Imam, N. Ramazani and M. R. Fayazi, J. Dent. (Tehran, Iran), 2015, 12, 430-435.

41. Z. Yingchao, G. Haihuan, F. Dan, F. Tengjiaozi, C. Danfeng, S. Zuosen, Z. Song and C. Zhanchen, J. Mater. Chem. B., 2015, 3, 4401-4405.

42. A. Domanska and A. Boczkowska, Polym. Degrad. Stab., 2014, 108, 175-181.
43. C. Cozean, C. J. Arcoria, J. Pelagalli and G. L. Powell, J. Am. Dent. Assoc., 1997, 128, 1080-1087.

44. J. Phanombualert, P. Chimtim, T. Heebthamai and W. Weera-Archakul, Photomed.Laser. Surg., 2015, 33, 467-472.

45. F. Shafiei and M. Memarpour, J. Dent.Child (Chic)., 2015, 82, 3-9.

46. G. Muhammed and R. Dayem, Lasers Med. Sci., 2015, 30, 969-975.

47. E. B. Tuna, E. Ozel, Y. Kasimoglu and E. Firatli, Microsc. Res. Tech., 2017, 80, 530-536.

48. E. Ozel, E. B. Tuna, S. Firatli and E. Firatli, Scanning, 2016, 38, 434-441.

49. S. Tao, L. Li, H. Yuan, S. Tao, Y. Cheng, L. He and J. Li, J. Evid. Based Dent. Pract., 2017, 17, 324-334.

50. R. M. Araujo, C. de Paula Eduardo, S. L. L. Duarte Junior, M. A. M. Araujo and L. de Castro Monteiro Loffredo, J. Clin. Laser. Med. Surg., 2001, 19, 325-332.

51. J. H. Chen, Y. Liu, L. N. Niu, S. Lu, F. R. Tay and Y. Gao, J. Adhes. Dent., 2014, 16, 429-434.

52. W. Zhou, L. N. Niu, L. Hu, K. Jiao, G. Chang, L. J. Shen, F. R. Tay and J. H. Chen, Sci. Rep., 2016, 6, 3274032746.

53. C. A. Carrera, C. Lan, D. Escobar-Sanabria, Y. Li, J. Rudney, C. Aparicio and A. Fok, Dent. Mater., 2015, 31, 382-390.

54. H. J. Kim and S. H. Park, Oper. Dent., 2014, 39, e57-e70.

55. X. Y. Zhao, S. B. Li, L. J. Gu and Y. Li, Oper. Dent., 2014, 39, 174-180.

56. S. Jacker-Guhr, G. Ibarra, L. S. Oppermann, A. K. Lührs, A. Rahman and W. Geurtsen, Clin. Oral Investig., 2016, 20, 1709-1718.

57. D. Huang, E. A. Swanson, C. P. Lin, J. S. Schuman, W. G. Stinson, W. Chang, M. R. Hee, T. Flotte, K. Gregory and C. A. Puliafito, Science, 1991, 254, 11781181.

58. S. H. Han, A. Sadr, J. Tagami and S. H. Park, Dent. Mater, 2016, 32, e1-e7.

59. C.-W. Sun, Y.-C. Ho and S.-Y. Lee, J. Sensors, 2015, 1-6, doi:10.1155/2015/984627. 
